\title{
Sex Differences and Olfactory Bulb Alterations Contribute to Limbic Circuit Dysfunction and Behavioral Disturbances in Pilocarpine Model of Epilepsy
}

Daniel Matovu ( $\nabla$ daniel.matovu@unifesp.br)

Escola Paulista de Medicina/UNIFESP

Esper A Cavalheiro

Escola Paulista de Medicina/UNIFESP

\section{Research Article}

Keywords: olfactory bulb, Sex differences, limbic circuit, dysfunction, pilocarpine model of epilepsy

Posted Date: June 22nd, 2021

DOI: https://doi.org/10.21203/rs.3.rs-624992/v1

License: (c) (1) This work is licensed under a Creative Commons Attribution 4.0 International License. Read Full License 


\section{Abstract}

The olfactory bulb at the sensory and circuit level transmits information to the limbic and cortical systems for behavioral outputs, and disruption of such circuits induces behavioral disturbances in rodents. Previously, data from our laboratory showed the occurrence of behavioral disturbances in Wistar rats submitted to the pilocarpine model of epilepsy (PME) and that these alterations were sex related. Here we deepen our findings that sex-linked differences are present in PME and that male epileptic rats exhibit profound recurrent seizure patterns, namely seizure duration, severity, and distribution along the light/dark cycle different from that observed in epileptic female rats. Further, using isotropic fractionator we observed significant alterations in the number of neuronal and non-neuronal cells of the olfactory bulb, amygdala, and hippocampus following 3 months of spontaneous recurrent seizures in epileptic male and female rats. Altogether, our study suggests that neuronal and non-neuronal cell death in olfactory bulb may interfere with sex-related differential recurrent seizure patterns, limbic circuit dysfunction, and behavioral disturbances in PME. Lastly, the pilocarpine epilepsy model provides an evidence-based tool to study mechanisms of behavioral disturbances in epileptogenesis that may provide future therapeutic insights in our quest to improve the life of people with epilepsy.

\section{Introduction}

Epilepsy is a brain disorder with electroclinical characteristics presenting recurrent non-evoked seizures, often associated with significant psychological morbidity and complications ${ }^{1,2}$. Comorbidities in epilepsy share complex and common pathogenic mechanisms whose pathophysiological mechanism is poorly understood and need to be studied as an essential part of epilepsy research ${ }^{3,4}$. Several studies on comorbidities related to epilepsy indicate that there are gender differences in these manifestations, for example, major depression is greater in female patients, while anxiety disorders are more common in men $^{5,6}$. Behavioral disturbances have also been studied in experimental epilepsy. Our group have observed that male and female rats submitted to the pilocarpine model of epilepsy present behavioral changes, such as: maternal behavior is disrupted in female epileptic rats that can even cannibalize their pups while male epileptic rats are aggressive and present hyposexuality 7,8 . Other behavioral abnormalities found in mice and rats submitted to the pilocarpine model include anxiety ${ }^{9,10}$, and depressive like behavior ${ }^{11,12}$.

Interestingly, similar behavioral disturbances and morphological alterations in the limbic circuits have also been observed in rats with olfactory bulbectomy ${ }^{13,14}$. The olfactory bulb of the rat is a crucial part of the neuronal network, whose sensory modality determines the immediate survival of an organism through activities such as the search for food, recognition of predators, social interaction, reproduction and several other aspects of behavior ${ }^{15}$. Neurons present in the olfactory bulb form diverse connections with various structures of the brain ${ }^{16}$. Via efferent connections to the amygdala and hippocampus, the olfactory bulb sends information to limbic structures where modulation and orchestration of emotional and behavioral response take place ${ }^{15}$. It is impressive to note the importance of olfactory stimuli to the 
relationship between rodent mothers and their offspring. In fact, extensive circuit reorganization and neurogenesis occur in the olfactory bulb and associated structures during pregnancy, delivery and puerperium of female rats and mice ${ }^{17,18}$.

Epileptic circuits, regardless of the underlying mechanism lead to an imbalance between glutamate and GABA activity ${ }^{19}$. This imbalance may be associated with circuit alterations that directly or indirectly contribute to limbic dysfunction and sex-linked behavioral disturbances observed in the pilocarpine epilepsy model.

Although the occurrence of psychiatric and behavioral comorbidities associated with epilepsy are well recognized ${ }^{20}$, the mechanisms underlying these comorbidities in humans and in animals with temporal lobe epilepsy are poorly understood.

Recurrent seizures are the hallmark of behavioral manifestation of temporal lobe epilepsy ${ }^{21}$. Studies in the pilocarpine model of epilepsy showed that limbic seizures commit to neuronal damage and circuit disruption ${ }^{22,23}$. Different lines of evidence reported that mechanistic characterization of recurrent seizures is poorly understood and lacking in sex difference studies of epilepsy ${ }^{24,25}$. Furthermore, we clearly do not understand, how neuronal death and injury-stimulated circuitry reorganization present in the epileptic brain, may induce behavioral disturbances in male and female rats.

The aim of the present study was to understand whether the occurrence of behavioral disturbances in male and female rats submitted to the pilocarpine model is related to different patterns of brain injuries involving the limbic system and to verify whether changes in the olfactory bulb are also sex related during epileptogenesis. Understanding modifications that occur in the olfactory bulb, amygdala and hippocampus underlying long-term recurrent seizures is important in the pathophysiology of temporal lobe epilepsy and could advance our understanding of epileptic brain pathways involved in associated comorbidities.

\section{Results}

Initiation of status epilepticus in all animals was observed at an average of 25 min after pilocarpine administration (range 15-37.8 minutes). Both male and female Wistar rats were exposed to $5 \mathrm{hr}$ of status epilepticus (SE) and were able to sustain seizure stage score of 3 or higher on the Racine scale. Unpaired t-test showed no statistical significance between the mean latent period (time in days between SE induction and expression of first spontaneous seizure) of epileptic males (15.1 days) and female rats (14.6 days), $p>0.627$. Graph not shown.

We examined male and female Wistar rats submitted to pilocarpine after the first spontaneous seizure using continuous video monitoring and quantified for recurrent seizure patterns using the Racine score scale. Repeated measures two-way ANOVA revealed significant main effect of sex $(F(1,8)=91.35 ; p<$ $0.0001)$, time $(F(1.301,10.41)=88.03 ; p<0.0001)$ and interaction between sex and time $(F(2,16)=$ 
31.60; $p$ < 0.0001) on seizure duration over a 3-month period. Sidak's post-hoc tests showed that the duration of each single seizure was higher in male in comparison to female epileptic rats in the1st (mean gap $=109.8 \mathrm{sec}, \mathrm{p}<0.003$ ), in the 2nd (mean gap $=274.8 \mathrm{sec}, \mathrm{p}<0.001)$, and in the 3rd month (mean gap $=610.4 \mathrm{sec}, \mathrm{p}<0.0008$ ) of observation (Fig. 1A).

On the other hand, and as shown in Fig. 1B, the number of spontaneous seizures in female epileptic rats was significantly higher than that observed in male epileptic rats during the 2 first months of observation $(F(1,8)=26.02 ; p<0.0009)$ for sex difference; $(F(1.973,15.79)=34.32 ; p<0.0001$ for month of observation]. Sidak's post-hoc tests revealed male epileptic rats had less spontaneous seizures when compared with female epileptic rats in the 1 st (mean gap $=7.2$ seizures, $p<0.0003$ ) and in the 2 nd month (mean gap $=4$ seizures, $p<0.004$ ) of observation. No difference in the number of seizures was observed during the 3rd month.

Figure 1C shows the significant effect of $\operatorname{sex}(F(1,8)=88.20 ; p<0.0001)$, of time $(F(1.985,15.88)=$ $25.57 ; p<0.0001)$ and the interaction between sex and time $(F(2,16)=4.696 ; P<0.02)$ in seizure severity. Sidak's post-hoc tests revealed that the severity of spontaneous seizures based on Racine scale ${ }^{26}$ in male epileptic rats was higher than that observed in female epileptic rats in the 1 st (mean range $=1, p<0.004$ ), in the 2 nd (mean range $=1.4, p<0.007$ ), and in 3rd month (mean range $=2, p<0.0003$ ).

The occurrence of cluster of seizures during the observation period showed also to be influenced by the $\operatorname{sex}(F(1,8)=43.20 ; P<0.0002)$ and by the time $(F(2,16)=4.098 ; p<0.03)$. Sidak's post-hoc tests showed that male epileptic rats had less seizure clusters during the first month than the female epileptic rats (mean range $=4$ seizure groups, $p<0.0001$ ). However, no difference was observed in the 2 nd and $3 r d$ month $(p>0.05)$ of observation (Fig. 1D).

Repeated measures two-way ANOVA demonstrated significant main effects of $\operatorname{sex} F(1,8)=27075 ; p<$ $0.0001)$, time $(F(1.393,11.14)=7411 ; p<0.0001)$ and interaction between sex and time $(F(11,88)=$ 37.23; $p<0.0001)$ in pattern of distribution of spontaneous recurrent seizures recorded during the light/dark cycle for a period of 3 months total. Sidak's post-hoc tests showed epileptic male rats had a significant increase in the number of seizures during light/dark cycles when compared with female epileptic rats throughout the 24 hours, $p<0.0001$.

Two-way ANOVA demonstrated a significant main effect of $\operatorname{sex}(F(1,16)=30.48 ; p<0.0001)$ but not group $(F(1,16)=1.976 ; p=0.1789)$ and interaction between sex and group $(F(1,16)=0.4245 ; p=$ 0.5240 ) in brain mass between control and male and female epileptic rats. Tukey's post-hoc tests showed significant decrease in brain mass between control male and epileptic male rats (mean gap $=0.14 \mathrm{~g}, \mathrm{p}<$ 0.0159 ), control female and epileptic female rats (mean gap $=0.18 \mathrm{~g}, \mathrm{p}<0.0024$ ) but no other significant group differences were observed $p>0.05$ (Fig. 3A).

Figure 3B shows that the hippocampal mass significantly decreased $(F(1,16)=27.38 ; p<0.0001)$ in epileptic animals (males or females) compared to control animals (males or females). However, the decrease in mass related to the presence of epilepsy was similar for animals of both sexes $(F(1,16)=$ 
$2420 ; p=0.1394)$, that is, the decrease in the epileptic hippocampus was $32 \%$ for males $(p<0.0173)$ and $33.3 \%$ for females $(p<0.0051)$ in relation to the respective controls. Figure $3 \mathrm{C}$ shows the amygdala mass in control animals and rats with epilepsy. It can be observed that the amygdala mass was significantly reduced $(F(1,16)=3.556 ; p=0.0002)$ in both male and female animals with epilepsy when compared to controls. However, when analyzing the loss of the amygdala mass between female and male rats with epilepsy, it is noted that the loss of mass of the amygdala in female rats with epilepsy (57.1\%) was greater than that observed in male rats with epilepsy (41.8\%).

In relation to the olfactory bulb (Fig. 3D), we could notice that a significant difference in the mass of this structure was already present between females and control males. The olfactory bulb in female rats is approximately $19.5 \%$ heavier than that of male rats $(p<0.02)$. In both groups, the presence of epilepsy significantly decreased olfactory bulb mass in animals of both sexes and, interestingly, mass loss was greater in male rats with epilepsy $(F(1,16)=44.31 ; p<0.0001)$ than in female rats with epilepsy $((F)(1$, 16) $=37.23 ; p<0.0001$ ).

Isotropic fractionator method was used to examine for the neuronal and non-neuronal cells in the hippocampus, amygdala and olfactory bulb respectively. Figures (4A), two-way ANOVA showed significant main effect of $\operatorname{sex}(F(1,16)=93.77 ; p<0.0001)$, group $(F(1,16)=38.60 ; p<0.0001)$ but not interaction between sex and group $(F(1,16)=4.23 ; p=0.0564)$ in the number of hippocampal neuronal cells. Tukey's post-hoc tests showed significant difference in the number of hippocampal neuronal cells between groups (control males and epileptic males with 29.8\% reduction, $\mathrm{p}<0.0001$ ), (control females and epileptic females with $17.5 \%$ reduction, $p<0.0003$ ), (epileptic males and epileptic females with $12.3 \%$ reduction, $p<0.0001$ ) and (control males and control females with $10.5 \%$ increase, $p<0.04$ ).

Figure 4B shows significant main effect of $\operatorname{sex}(F(1,16)=136.7 ; p<0.0001)$, interaction between sex and group $(F(1,16)=18.20)$ but not group $(F(1,16)=2.49 ; p=0.1337)$ in the number of amygdala neuronal cells. Tukey's post-hoc tests showed significant decrease in the number of amygdala neuronal cell between groups (control males and epileptic males with $17.7 \%$ reduction, $p<0.0004$ ), (control females and epileptic females with $35.8 \%$ reduction, $p<0.0001$ ), (epileptic males and epileptic females with $18.1 \%$ reduction, $p<0.0003$ ) but no differences were observed between other groups.

Figure (4C), the counting of neuronal cells in the olfactory bulb revealed significant main effect of sex ( $\mathrm{F}$ $(1,16)=126.8 ; p<0.0001)$, of group $(F(1,16)=47.64 ; p<0.0001)$ but not of interaction between sex and group $(F(1,16)=1.31 ; p=0.2681)$. Tukey's post-hoc tests showed significant difference in the number of olfactory bulb neuronal cells between groups (control males and epileptic males with $27 \%$ reduction, $p<$ 0.0001), (control females and epileptic females with $19.5 \%$ reduction, $p<0.0001$ ), (epileptic males and epileptic females with $7.5 \%$ reduction, $p<0.0002$ ) and (control males and females with $12.5 \%$ increase, $p$ $<0.004)$.

Regarding the non-neuronal cells present in the three analyzed structures (Fig. 5A), the two-way ANOVA test showed a significant variation in the number of these cells depending on the group (control versus epilepsy; $F(1,16)=72.38 ; p<0.0001)$, of the interaction between sex and group $(F(1,16)=7.66 ; p=$ 
$0.0137)$, but not $\operatorname{sex}(F(1,16)=2.35 ; P=0.1443)$. Tukey's post-hoc tests showed a significant increase in the number of non-neuronal cells between groups (control female rats versus female rats with epilepsy with $10 \%$ increase, $p<0.03$ ), (control male rats versus female control rats with $17 \%$ increase, $p<0.004$ ), (epileptic male rats versus epileptic female rats with $36.1 \%$ increase, $p<0.0001$ ), but no differences were observed between the other groups.

The number of non-neuronal cells in the amygdala (Fig. 5B) varied significantly according to the group (control animals versus animals with epilepsy; $F(1,16)=43.28 ; p<0.0001$ ), and when analyzing the interaction between groups and $\operatorname{sex}(F(1,16)=13.50 ; P=0.0021)$, but there was no significant difference when the only variable considered was the sex of the animals $(F(1,16)=3.21 ; P=0.0917)$. Tukey's posthoc tests showed a significant increase in the number of non-neuronal tonsil cells between groups (control female rats and female rats with epilepsy with an increase of $7.4 \%, p<0.006$ ) and (male rats with epilepsy versus female rats with epilepsy with increase of $14.7 \%, p<0.0001)$. No significant differences were observed between the other groups.

For the olfactory bulb (Fig. 5C), we observed a predominant effect of $\operatorname{sex}(F(1,16)=5.15 ; p=0.0374)$, from the group $(F(1,16)=183.6 ; p<0.0001)$ and from the interaction between sex and group $(F(1,16)=$ $11.32 ; p=0.0039)$ on the number of non-neuronal cells. Tukey's post-hoc tests showed a significant increase $(9.9 \%, p<0.005)$ in the number of non-neuronal cells in the olfactory bulb of female rats with epilepsy when compared to values obtained in the olfactory bulb of control female rats. We did not observe statistical differences when this analysis was performed comparing the number of non-neuronal cells present in the olfactory bulb of male control rats with the values found in male rats with epilepsy ( $p$ $>0.864$ ). Another important finding observed in the study of the olfactory bulb was the greater number $(37 \%, p<0.0001)$ of non-neuronal cells present in female rats with epilepsy when compared to that found in males with epilepsy

\section{Discussion}

The present study provides two main novel findings in the epileptic brain. First, significant differences in the spontaneous recurrent seizure patterns exist among male and female rats submitted to the pilocarpine model of epilepsy. Second, the topographic distribution of neuronal and non-neuronal cell death differs in the brains of male and female epileptic rats. These findings provide one possible explanation for limbic circuit dysfunction and behavioral disturbances observed in the pilocarpine epilepsy model. There is scanty data on chronic epileptic seizure patterns in female rats, most of the information is tailored towards S.E which does not meet the comparison criteria.

The present evidence summarizes significant sex-linked differences in main structures and long term recurrent seizure patterns: male and female rats demonstrated differences in amygdala and olfactory bulb mass reduction, hippocampus, amygdala and olfactory neuronal loss and non-neuronal cell death. Additionally, epileptic male rats exhibited increased seizure duration, severity and more seizures during 
light/dark cycles per day while epileptic females showed increased seizure frequency and seizure clusters when compared during the 3 months period.

It is important to note the pilocarpine epilepsy model is an appropriate paradigm to study extensive extra hippocampal lesions or adjacent structure involvement in temporal lobe epilepsy ${ }^{27,28}$. Pilocarpineinduced neuronal death in limbic circuitry follows a cascade of events: from the activation of muscarinic receptors to the over activation of AMPA and NMDA receptors culminating in massive permeability to calcium ions leading to neuronal injury and death. Insights on how glutamatergic pathways promote hyperexcitable circuits associated with neuronal alterations and damage to adjacent structures has been provided by kindling studies ${ }^{29}$. For instance, olfactory bulb kindling facilitates spread of seizure activity via extensive connections to the amygdala and to the hippocampus leading to permanent structural changes in these structures ${ }^{30}$. Additionally, repeated amygdaloid kindling results into profound seizure activity and synaptic reorganization of the olfactory bulb-subventricular zone ${ }^{31}$.

The different distribution of neuronal and non-neuronal cell death in the limbic system of male and female epileptic rats may reflect the differential distribution of receptors to sex hormones in these structures as well as the connections formed between them throughout life and other factors such as the circadian cycle. The fact that female rats express higher density and differential distribution of estrogen receptors in the limbic structures compared to male rats and the influence of the estrous cycle and circadian cycle in the number and function of these receptors is well known ${ }^{32}$.

In relation to experimental epilepsy, our laboratory has previously shown that epileptic female rats release increased estradiol and reduced progesterone levels ${ }^{33}$. Epileptic female rats are susceptible to progesterone withdrawal predisposing to increased seizure frequency and decreased allopregnanolone levels have been implicated in this phenomenon ${ }^{25}$. The mechanisms by which these two hormones promote or hinder seizure activity in limbic circuits are not fully understood in the brain ${ }^{33}$. In brief within brain circuits, estradiol is bidirectional ligand that exerts its proconvulsant effects through NMDA receptor and anticonvulsant action by Neuropeptide $Y$ within the brain ${ }^{34,35}$. Progesterone mediates its anticonvulsant through allopregnanolone ${ }^{36,37}$. Testosterone is converted to estradiol promoting intense tonic clonic seizures observed in male epileptic rats ${ }^{36,38}$. Furthermore, progesterone has shown to modulate neuronal and non-neuronal cell death, neuroinflammation, neurogenesis in epileptic female rats $^{39}$.

In support with our findings (Figs. 1 and 2), kainic and pilocarpine model of epilepsy agree that epileptic male rats gradually intensify seizures characterized with increase in duration, severity and frequency with more seizure occurring during the light cycles of day over a period of time ${ }^{40-42}$. Seizure clusters have been identified in sub population of animals as seen in humans with TLE but exact mechanisms by which dysfunctional microcircuits trigger, propagate and terminate while expressing these different seizure patterns remain elusive ${ }^{41}$. We have earlier demonstrated that epileptic male rats continue to lose neurons in the hippocampus as they also exhibited recurrent seizures throughout their life-time ${ }^{43}$. 
Brought together, sex differences and broad mechanistic effects of gonadal hormones on limbic excitability and seizure vulnerability may be influenced by various factors like dimorphic disparities in epileptogenic regions of the brain that initiate and terminate seizures, intrinsic brain connectivity, receptor distribution and signaling pathways ${ }^{44}$. These changes may contribute to the differences in recurrent seizure patterns including seizure frequency, duration, clusters and severity.

Sex-linked differences of neuronal and non-neuronal cell death seen in the olfactory bulb, amygdala and hippocampus happens in a very important pathway that modulate behavioral outputs and also form a sensory window to the outside environment in rats. The long term recurrent seizures promote gradual neuron loss disrupting limbic circuits throughout epilepsy ${ }^{43}$. For instance, several lines of evidences in experimental epilepsy reported GABAergic circuit disruption of parvalbumin cell death in the olfactory bulb $^{45}$, basolateral amygdala ${ }^{29}$, and hippocampus ${ }^{46}$. Via neural pathways, ventral CA1-hippocampal region and basolateral amygdala heavily rely on the olfactory sensory inputs to modulate fear, mood and anxiety-like behaviors ${ }^{47}$. In addition, these regions also express the greatest cell loss and damage from epileptic seizures 29,46 , and contain anxiety circuit pathways to the prefrontal cortex and hypothalamus ${ }^{48,49}$. In the face of temporal lobe epilepsy, olfactory glutamatergic dysfunction via pathways may promote hyperexcitability in epileptogenic regions of the amygdala and hippocampus eliciting more recurrent seizures, damaging microcircuits that control behavior within limbic system and cortical connections.

Therefore, we conclude that sex-linked differences and recurrent seizure patterns in temporal lobe epilepsy signify the extent of brain damage and these microcircuit damages through neuronal death within the limbic system may contribute behavioral disturbances observed in the pilocarpine epilepsy model. Our results suggest that neuronal and non-neuronal cell death with differential recurrent seizure patterns among epileptic male and female rats may contribute to limbic circuit dysfunction and behavioral disturbances in the pilocarpine model of epilepsy. We have begun to explore genetic manipulations and special cell types in the olfactory-limbic pathways involved in circuit-level dysfunction of behavior disturbances in TLE. We hope that our studies will help identify potential molecular mechanisms and targets for behavioral disturbances in order to bridge our understanding of epilepsy within brain circuits.

\section{Methods}

\section{Ethical declaration}

This study was approved by the Ethic Committee on Animal Use of the Federal University of Sao Paulo (CEUA/UNIFESP) under the reference number 8624210118. All animal experiments were in accordance to international guidelines of animal care ${ }^{50}$ and ARRIVE guidelines for conducting invivo experiments of animal research ${ }^{51}$.

\section{Animals and Experimental groups}


A total of 30 Wistar rats were used, consisting of males and female rats weighing 210 to $245 \mathrm{~g}$ that were grouped into 4 groups namely: control males $(n=5)$, control females $(n=5)$, epileptic males $(n=10)$ and epileptic females $(n=10)$ respectively. Animals were housed in groups of four in controlled climatic conditions (light-dark cycle of 12 hours, lights on between 7 AM and 7 PM, and constant temperature of $\left(19-23^{\circ} \mathrm{C}\right)$, with free access to water and food. All experiments were randomly grouped, and a blinded manner approach used until data was analyzed.

\section{Vaginal Cytology}

Female Wistar rats were introduced with noninvasive dropper to obtain vaginal contents for examination under the microscope. The vaginal specimen was collected every $8 \mathrm{AM}$ and $10 \mathrm{AM}$ to observe for estrous cycle pattern following an interval of 3 to 4 days. Only animals with estrous phase pattern were included in the study and submitted to pilocarpine administration ${ }^{52}$.

\section{Pilocarpine Epilepsy Model}

The pilocarpine model of epilepsy represents a suitable paradigm to study pathophysiological mechanisms of temporal lobe epilepsy and has been used in numerous studies to the phenomenon ${ }^{27}$. Shortly, female Wistar rats in the estrus stage and males were pretreated with methyl scopolamine (Sigma Co.) at dose of $1 \mathrm{mg} / \mathrm{kg}$ subcutaneously to inhibit systemic cholinergic effects of pilocarpine 30 minutes prior ${ }^{33}$. Thereafter, pilocarpine hydrochloride (Sigma Co.) at a dose of $330 \mathrm{mg} / \mathrm{kg}$ was injected intraperitoneally to induce progressive sequential behavioral and electrographic changes leading to status epilepticus of long duration ${ }^{22,33}$. After five hours of status epilepticus graded on the Racine scale, diazepam (Merck) at a dose of $10 \mathrm{mg} / \mathrm{kg}$ was given subcutaneously to limit behavioral limbic seizures ${ }^{53}$. The surviving animals ( 7 males and 9 females) were nursed with a special fractionated diet till recovery and transferred to the video lab for monitoring. Control male and female rats were injected with methyl scopolamine ( $1 \mathrm{mg} / \mathrm{kg}$, s.c.) followed 30 minutes later by saline solution $(1 \mathrm{ml} / \mathrm{kg}$, i.p.) instead pilocarpine.

\section{Seizure Video Monitoring}

A high-definition video system (Intelbras VT 4 S 120 HD) was used to continuously monitor the pilocarpine treated animals for recurrent seizure activity for $24 \mathrm{~h}$ every day 43 . Limbic seizure behavior was monitored for three months after the first spontaneous seizure (SRS) under the following parameters: number, duration, and severity of recurrent seizures. Animals surviving the pilocarpineinduced status epilepticus evolved into the period of spontaneous recurrent seizures of the epilepsy model. Seizure distribution in 24h was recorded for light (07:00-18:59h) and dark (19:00-06:59h) periods. All seizure parameters were analyzed according to the progressive scale proposed by Racine for the amygdala kindling ${ }^{26}$, as follows: 1 . mouth and facial movements; 2 . head nodding; 3. forelimb clonus; 4. forelimb clonus with rearing; 5 . rearing and falling. Seizure cluster was considered if an animal had $\geq 2$ seizures in $24 \mathrm{~h}^{54}$. 


\section{Isotropic fractionator method}

This method is used to quantify the number of neuronal and non-neuronal cells in the brain or dissected structure and has been compared in several studies with unbiased stereological techniques, producing reliable and reproducible results faster. This technique does not need special software but follows the similar principles used in stereological analysis to produce about $100 \%$ of the number of cells in the brain $^{55}$.

Briefly, at the end of the 3-month observation period, all animals from each group were thoroughly anesthetized with sodium thiopental $(80 \mathrm{mg} / \mathrm{kg}, \mathrm{sc}-$ Thiopentax) and perfused transcardially with phosphate-buffered solution (0.01 M - pH7.4), and thereafter, paraformaldehyde at $4 \%$ in PBS. Their brains were carefully removed and post fixed in paraformaldehyde at $4 \%$ for 24 hours. Then brains were separated into the three structures of interest: olfactory bulb, amygdala and hippocampal formation using rat brain matrices ( $1 \mathrm{~mm}$, Kent) fixed on crushed ice, and consistent anatomical landmarks under a Leica stereomicroscope. Olfactory bulbs were separated by cutting away from the frontal poles. Hippocampal formation was carefully dissected from each hemisphere by detaching it from the striatum and cortical tissue. Amygdala were identified lateral to both hypothalamus and ventral portion of the rhinal sulcus, by cutting away $8 \mathrm{~mm}$ of loose cortex allowed isolation of amygdala consisting of basolateral and centromedial portions of the structure ${ }^{56}$. Each brain structure was mechanically dissociated in a saline solution with $1 \%$ Triton X-100 and turned into an isotropic suspension of isolated nuclei, kept homogeneous by agitation. The total number of cells were estimated by determining the number of nuclei in small aliquots stained with fluorescent DNA marker 4'-6-diamidino-2-phenylindole dihydrochloride (DAPI, 1:1000; Sigma-Aldrich, D9542) utilizing a Zeiss Axiovert 100 microscope with a 40x objective, using a hemocytometer for quantification (Neubauer chamber, Loptik Labor). To determine neuronal and non-neuronal cells number, the samples were then incubated with the primary antibody against the neuron-specific nuclear protein (NeuN, 1:100; Abcam, ab104225) at $4{ }^{\circ} \mathrm{C}$ overnight and, subsequently, with secondary antibody conjugated to AlexaFluor ${ }^{\circledR} 488$ (Abcam; ab150077) diluted in PBS (1:200) and 10\% normal goat serum (Vector labs, S-1000-20) for $2 \mathrm{~h}$. The neuronal fraction in each sample was estimated by counting NeuN-labeled nuclei in at least 500 DAPI-stained nuclei and the number of non-neuronal nuclei were obtained by subtraction ${ }^{57}$.

\section{Statistical Analysis}

Shapiro-Wilk test was done to ascertain data normality using Graph Pad Prism version 8.4.2 (Graph Pad Software, La Jolla California USA). Unpaired t-test was used to analyze for the duration of latent period in pilocarpine treated animals. Two-ANOVA (repeated measures) and Sidak's post-hoc test was performed for seizure dependent variables. Independent two-way ANOVA and Tukey's post-hoc test were carried out on dependent variables: mass, number of neurons and non-neuronal cell of brain structures. Data was presented as means and standard deviation with level of significance set at 0.05 .

\section{Declarations}




\section{Acknowledgements}

This work was supported by research grant from CNPq, Brazil (to EAC) and an Institutional research grant to National Institute of Translational Neuroscience (MCTIC, CNPq and FAPERJ of Brazil). This study was financed in part by the Coordenaçāo de Aperfeiçoamento de Pessoal de Nível Superior - Brasil (CAPES) - Finance Code 001. We thank: Dr Sandra R Perosa, Dr Simone Bittencourt, Daniel Pinheiro and Maria Marques for their technical and statistical assistance with this project.

\section{Contributions}

Authors contributions were as follows: D.M and E.A.C. designed the research, D.M. performed the pilocarpine epilepsy experiments, D.M. and E.A.C. conducted the isotropic fractionator and neuronal quantification experiments, D.M and E.A.C. analyzed the data; D.M and E.A.C. wrote the paper.

\section{Competing Interests}

Authors declare no competing interests.

\section{References}

1. Devinsky, O. et al. Epilepsy. Nature Reviews Disease Primers 4, 18024, doi:10.1038/nrdp.2018.24 (2018).

2. Goldberg, E. M. \& Coulter, D. A. Mechanisms of epileptogenesis: a convergence on neural circuit dysfunction. Nature reviews. Neuroscience 14, 337-349, doi:10.1038/nrn3482 (2013).

3. Kanner, A. M., Ribot, R. \& Mazarati, A. Bidirectional relations among common psychiatric and neurologic comorbidities and epilepsy: Do they have an impact on the course of the seizure disorder? Epilepsia open 3, 210-219, doi:10.1002/epi4.12278 (2018).

4. Mazarati, A. Can we and should we use animal models to study neurobehavioral comorbidities of epilepsy? Epilepsy \& behavior : E\&B 101, 106566, doi:10.1016/j.yebeh.2019.106566 (2019).

5. Gaus, V., Kiep, H., Holtkamp, M., Burkert, S. \& Kendel, F. Gender differences in depression, but not in anxiety in people with epilepsy. Seizure 32, 37-42, doi:10.1016/j.seizure.2015.07.012 (2015).

6. Liu, Z. et al. Gender Differences in Associated and Predictive Factors of Anxiety and Depression in People With Epilepsy. Frontiers in psychiatry 11, 670, doi:10.3389/fpsyt.2020.00670 (2020).

7. Alvarenga, T. A. et al. Sexual response in female rats with status epilepticus. Epilepsia 54, 644-648, doi:10.1111/epi.12117 (2013).

8. Lima, D. C. et al. Behavioral evaluation of adult rats exposed in utero to maternal epileptic seizures. Epilepsy \& behavior : E\&B 18, 45-49, doi:10.1016/j.yebeh.2010.02.024 (2010).

9. Gröticke, I., Hoffmann, K. \& Löscher, W. Behavioral alterations in the pilocarpine model of temporal lobe epilepsy in mice. Experimental neurology 207, 329-349, doi:10.1016/j.expneurol.2007.06.021 (2007). 
10. Lopes, M. W. et al. Time course evaluation of behavioral impairments in the pilocarpine model of epilepsy. Epilepsy \& behavior : E\&B 55, 92-100, doi:10.1016/j.yebeh.2015.12.001 (2016).

11. Mazarati, A. et al. Depression after status epilepticus: behavioural and biochemical deficits and effects of fluoxetine. Brain : a journal of neurology 131, 2071-2083, doi:10.1093/brain/awn117 (2008).

12. Peng, W. F. et al. N-methyl-D-aspartate receptor NR2B subunit involved in depression-like behaviours in lithium chloride-pilocarpine chronic rat epilepsy model. Epilepsy research 119, 77-85, doi:10.1016/j.eplepsyres.2015.09.013 (2016).

13. Jindal, A., Mahesh, R. \& Bhatt, S. Etazolate, a phosphodiesterase-4 enzyme inhibitor produces antidepressant-like effects by blocking the behavioral, biochemical, neurobiological deficits and histological abnormalities in hippocampus region caused by olfactory bulbectomy. Psychopharmacology 232, 623-637, doi:10.1007/s00213-014-3705-0 (2015).

14. Yuan, M. Y. et al. Ablation of olfactory bulb glutamatergic neurons induces depressive-like behaviors and sleep disturbances in mice. 237, 2517-2530, doi:10.1007/s00213-020-05552-6 (2020).

15. Ennis, M., Puche, A. C., Holy, T. \& Shipley, M. T. in The Rat Nervous System (Fourth Edition) (ed George Paxinos) 761-803 (Academic Press, 2015).

16. McGann, J. P. Poor human olfaction is a 19th-century myth. Science (New York, N. Y.) 356, doi:10.1126/science.aam7263 (2017).

17. Belnoue, L., Malvaut, S., Ladevèze, E., Abrous, D. N. \& Koehl, M. Plasticity in the olfactory bulb of the maternal mouse is prevented by gestational stress. Scientific reports 6, 37615, doi:10.1038/srep37615 (2016).

18. Leuner, B. \& Sabihi, S. The birth of new neurons in the maternal brain: Hormonal regulation and functional implications. Frontiers in neuroendocrinology 41, 99-113, doi:10.1016/j.yfrne.2016.02.004 (2016).

19. Paz, J. T. \& Huguenard, J. R. Microcircuits and their interactions in epilepsy: is the focus out of focus? Nature neuroscience 18, 351-359, doi:10.1038/nn.3950 (2015).

20. Mula, M. The comorbidities of epilepsy explained. Expert review of neurotherapeutics 20, 1207-1209, doi:10.1080/14737175.2020.1840979 (2020).

21. Cersósimo, R. et al. Mesial temporal lobe epilepsy with hippocampal sclerosis: study of 42 children. Seizure 20, 131-137, doi:10.1016/j.seizure.2010.11.002 (2011).

22. Turski, W. A. et al. Limbic seizures produced by pilocarpine in rats: behavioural, electroencephalographic and neuropathological study. Behavioural brain research 9, 315-335, doi:10.1016/0166-4328(83)90136-5 (1983).

23. Wulsin, A. C. et al. Functional disruption of stress modulatory circuits in a model of temporal lobe epilepsy. PloS one 13, e0197955, doi:10.1371/journal.pone.0197955 (2018).

24. Samba Reddy, D. Sex differences in the anticonvulsant activity of neurosteroids. Journal of neuroscience research 95, 661-670, doi:10.1002/jnr.23853 (2017). 
25. Scharfman, H. E. \& MacLusky, N. J. Sex differences in the neurobiology of epilepsy: a preclinical perspective. Neurobiology of disease 72 Pt B, 180-192, doi:10.1016/j.nbd.2014.07.004 (2014).

26. Racine, R. J. Modification of seizure activity by electrical stimulation. II. Motor seizure. Electroencephalography and clinical neurophysiology 32, 281-294, doi:10.1016/00134694(72)90177-0 (1972).

27. Curia, G., Longo, D., Biagini, G., Jones, R. S. \& Avoli, M. The pilocarpine model of temporal lobe epilepsy. Journal of neuroscience methods 172, 143-157, doi:10.1016/j.jneumeth.2008.04.019 (2008).

28. Rusina, E., Bernard, C. \& Williamson, A. The Kainic Acid Models of Temporal Lobe Epilepsy. eneuro 8, ENEURO.0337-0320.2021, doi:10.1523/eneuro.0337-20.2021 (2021).

29. Aroniadou-Anderjaska, V., Fritsch, B., Qashu, F. \& Braga, M. F. M. Pathology and pathophysiology of the amygdala in epileptogenesis and epilepsy. Epilepsy research 78, 102-116, doi:10.1016/j.eplepsyres.2007.11.011 (2008).

30. Baimbridge, K. G., Mody, I. \& Miller, J. J. Reduction of rat hippocampal calcium-binding protein following commissural, amygdala, septal, perforant path, and olfactory bulb kindling. Epilepsia 26, 460-465, doi:10.1111/j.1528-1157.1985.tb05681.x (1985).

31. Sato, K., Iwai, M., Nagano, I., Shoji, M. \& Abe, K. Expression of highly polysialylated neural cell adhesion molecule in rat subventricular zone with exposure to repeated kindled seizures. Neuroscience letters 323, 244-246, doi:10.1016/s0304-3940(02)00139-8 (2002).

32. Zhang, J. Q., Cai, W. Q., Zhou, D. S. \& Su, B. Y. Distribution and differences of estrogen receptor beta immunoreactivity in the brain of adult male and female rats. Brain research $935,73-80$, doi:10.1016/s0006-8993(02)02460-5 (2002).

33. Amado, D. \& Cavalheiro, E. A. Hormonal and gestational parameters in female rats submitted to the pilocarpine model of epilepsy. Epilepsy research 32, 266-274, doi:10.1016/s0920-1211(98)00057-6 (1998).

34. Ledoux, V. A., Smejkalova, T., May, R. M., Cooke, B. M. \& Woolley, C. S. Estradiol Facilitates the Release of Neuropeptide $Y$ to Suppress Hippocampus-Dependent Seizures. The Journal of Neuroscience 29, 1457-1468, doi:10.1523/jneurosci.4688-08.2009 (2009).

35. Velísková, J. \& Velísek, L. Beta-estradiol increases dentate gyrus inhibition in female rats via augmentation of hilar neuropeptide Y. The Journal of neuroscience : the official journal of the Society for Neuroscience 27, 6054-6063, doi:10.1523/jneurosci.0366-07.2007 (2007).

36. Frye, C. A., Rhodes, M. E., Walf, A. \& Harney, J. Progesterone reduces pentylenetetrazol-induced ictal activity of wild-type mice but not those deficient in type I 5alpha-reductase. Epilepsia $\mathbf{4 3}$ Suppl 5, 1417, doi:10.1046/j.1528-1157.43.s.5.19.x (2002).

37. Reddy, D. S. Neurosteroids: endogenous role in the human brain and therapeutic potentials. Progress in brain research 186, 113-137, doi:10.1016/b978-0-444-53630-3.00008-7 (2010).

38. Teyler, T. J., Vardaris, R. M., Lewis, D. \& Rawitch, A. B. Gonadal steroids: effects on excitability of hippocampal pyramidal cells. Science (New York, N.Y.) 209, 1017-1018, 
doi:10.1126/science.7190730 (1980).

39. Reddy, D. S. Role of hormones and neurosteroids in epileptogenesis. Frontiers in cellular neuroscience 7, 115, doi:10.3389/fncel.2013.00115 (2013).

40. Arida, R. M., Scorza, F. A., Peres, C. A. \& Cavalheiro, E. A. The course of untreated seizures in the pilocarpine model of epilepsy. Epilepsy research 34, 99-107, doi:10.1016/s0920-1211(98)00092-8 (1999).

41. Goffın, K., Nissinen, J., Van Laere, K. \& Pitkänen, A. Cyclicity of spontaneous recurrent seizures in pilocarpine model of temporal lobe epilepsy in rat. Experimental neurology 205, 501-505, doi:10.1016/j.expneurol.2007.03.008 (2007).

42. Upadhya, D. et al. A Model of Chronic Temporal Lobe Epilepsy Presenting Constantly Rhythmic and Robust Spontaneous Seizures, Co-morbidities and Hippocampal Neuropathology. Aging and disease 10, 915-936, doi:10.14336/ad.2019.0720 (2019).

43. Lopim, G. M. et al. Relationship between seizure frequency and number of neuronal and nonneuronal cells in the hippocampus throughout the life of rats with epilepsy. Brain research 1634, 179186, doi:10.1016/j.brainres.2015.12.055 (2016).

44. Velíšková, J. \& Desantis, K. A. Sex and hormonal influences on seizures and epilepsy. Hormones and behavior 63, 267-277, doi:10.1016/j.yhbeh.2012.03.018 (2013).

45. Yu, Y. H., Park, D.-K., Yoo, D. Y. \& Kim, D.-S. Altered expression of parvalbumin immunoreactivity in rat main olfactory bulb following pilocarpine-induced status epilepticus. BMB Rep 53, 234-239, doi:10.5483/BMBRep.2020.53.4.002 (2020).

46. Yi, F. et al. Vulnerability of calbindin-positive interneurons to status epilepticus varies in different regions of rat hippocampus. Neurochemical Journal 8, 306-310, doi:10.1134/S1819712414040126 (2014).

47. Pi, G. et al. Posterior basolateral amygdala to ventral hippocampal CA1 drives approach behaviour to exert an anxiolytic effect. Nature communications 11, 183, doi:10.1038/s41467-019-13919-3 (2020).

48. Jimenez, J. C. et al. Anxiety Cells in a Hippocampal-Hypothalamic Circuit. Neuron 97, 670-683.e676, doi:10.1016/j.neuron.2018.01.016 (2018).

49. Parfitt, G. M. et al. Bidirectional Control of Anxiety-Related Behaviors in Mice: Role of Inputs Arising from the Ventral Hippocampus to the Lateral Septum and Medial Prefrontal Cortex.

Neuropsychopharmacology : official publication of the American College of Neuropsychopharmacology 42, 1715-1728, doi:10.1038/npp.2017.56 (2017).

50. National Research Council Committee for the Update of the Guide for the, C. \& Use of Laboratory, A. in Guide for the Care and Use of Laboratory Animals (National Academies Press (US)Copyright (C) 2011, National Academy of Sciences., 2011).

51. Percie du Sert, N. et al. The ARRIVE guidelines 2.0: Updated guidelines for reporting animal research. British journal of pharmacology 177, 3617-3624, doi:10.1111/bph.15193 (2020).

52. do Vale, T. G. et al. Seizures during pregnancy modify the development of hippocampal interneurons of the offspring. Epilepsy \& behavior : E\&B 19, 20-25, doi:10.1016/j.yebeh.2010.06.032 (2010). 
53. Lemos, T. \& Cavalheiro, E. A. Suppression of pilocarpine-induced status epilepticus and the late development of epilepsy in rats. Experimental brain research 102, 423-428, doi:10.1007/bf00230647 (1995).

54. Jafarpour, S., Hirsch, L. J., Gaínza-Lein, M., Kellinghaus, C. \& Detyniecki, K. Seizure cluster: Definition, prevalence, consequences, and management. Seizure 68, 9-15, doi:10.1016/j.seizure.2018.05.013 (2019).

55. Herculano-Houzel, S., von Bartheld, C. S., Miller, D. J. \& Kaas, J. H. How to count cells: the advantages and disadvantages of the isotropic fractionator compared with stereology. Cell and tissue research 360, 29-42, doi:10.1007/s00441-015-2127-6 (2015).

56. Gilbert, K., Godbout, R. \& Rousseau, G. Caspase-3 Activity in the Rat Amygdala Measured by Spectrofluorometry After Myocardial Infarction. Journal of visualized experiments : JoVE, e53207, doi:10.3791/53207 (2016).

57. Herculano-Houzel, S., Mota, B. \& Lent, R. Cellular scaling rules for rodent brains. Proceedings of the National Academy of Sciences of the United States of America 103, 12138-12143, doi:10.1073/pnas.0604911103 (2006).

\section{Figures}
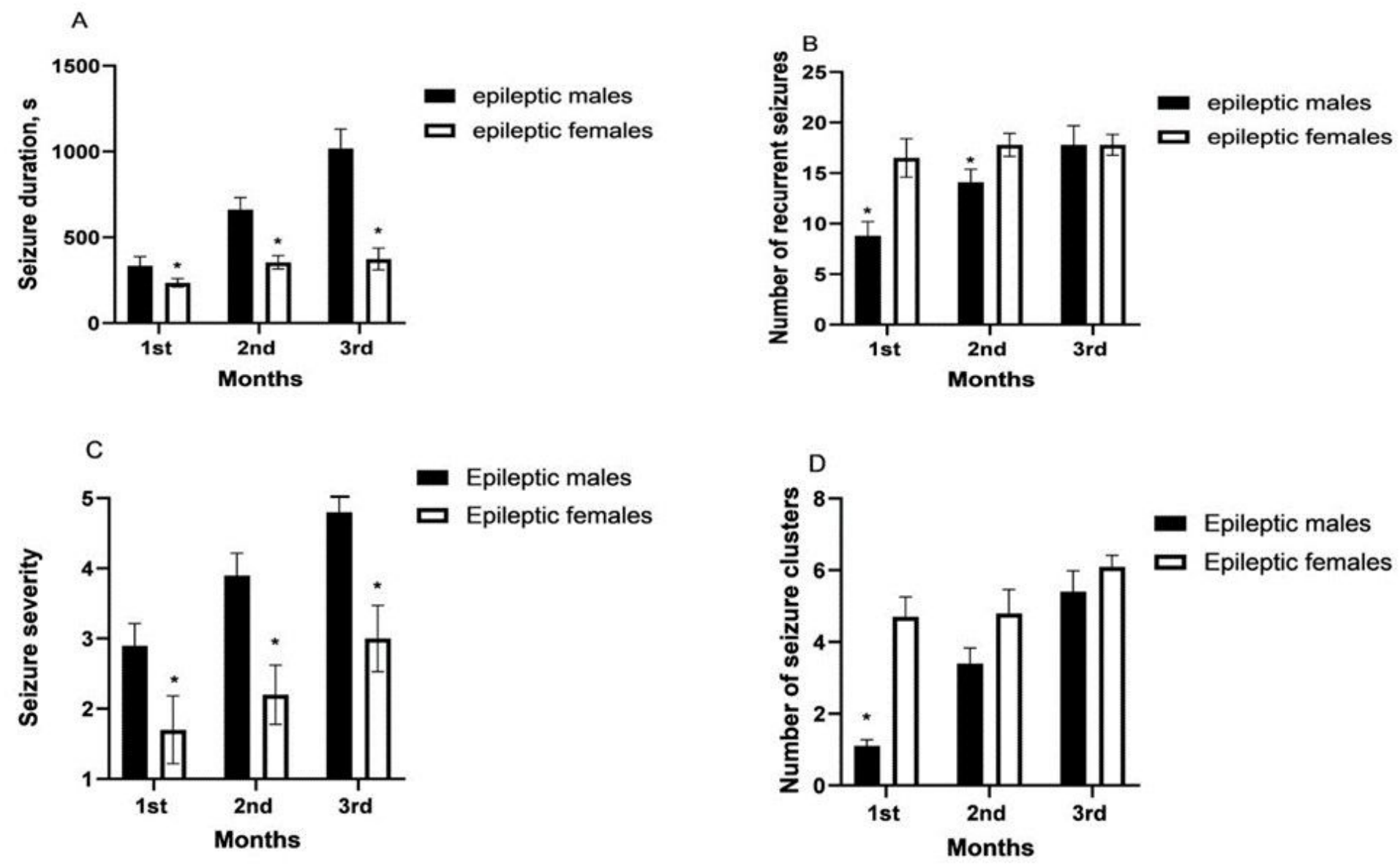

Figure 1 
Characterization of recurrent seizure patterns in both epileptic male and female rats submitted to $5 \mathrm{~h}$ of status epilepticus (SE) and monitored continuously $24 \mathrm{~h}$ for a period of 3 months after expression of the first spontaneous seizure. (A) Seizure duration in seconds (s). (B) Number of recurrent seizures. (C) Seizure severity score according to the Racine scale (see Methods). (D) Number of seizure clusters. Data expressed as mean \pm standard deviation. ${ }^{*} p<0.05$ for signifance.

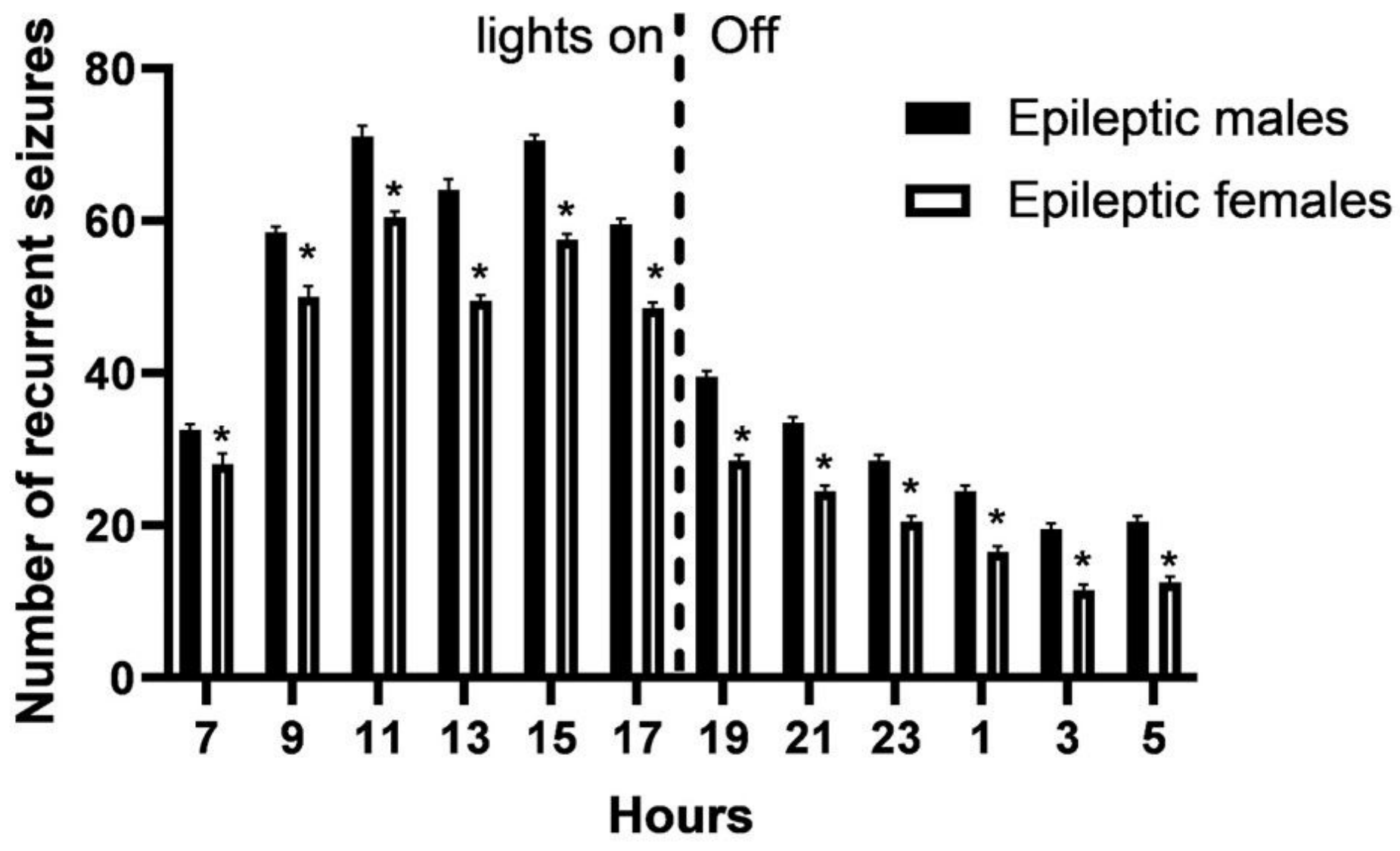

Figure 2

Recurrent seizure distribution in $2 \mathrm{~h}$ pattern for $24 \mathrm{~h}$ recordings over a period 3 months during the light (07:00-18:59h) and dark (19:00-06:59h) cycle in both male and female epileptic rats. Data expressed as mean \pm standard deviation. ${ }^{*} \mathrm{p}<0.05$ for significance. 

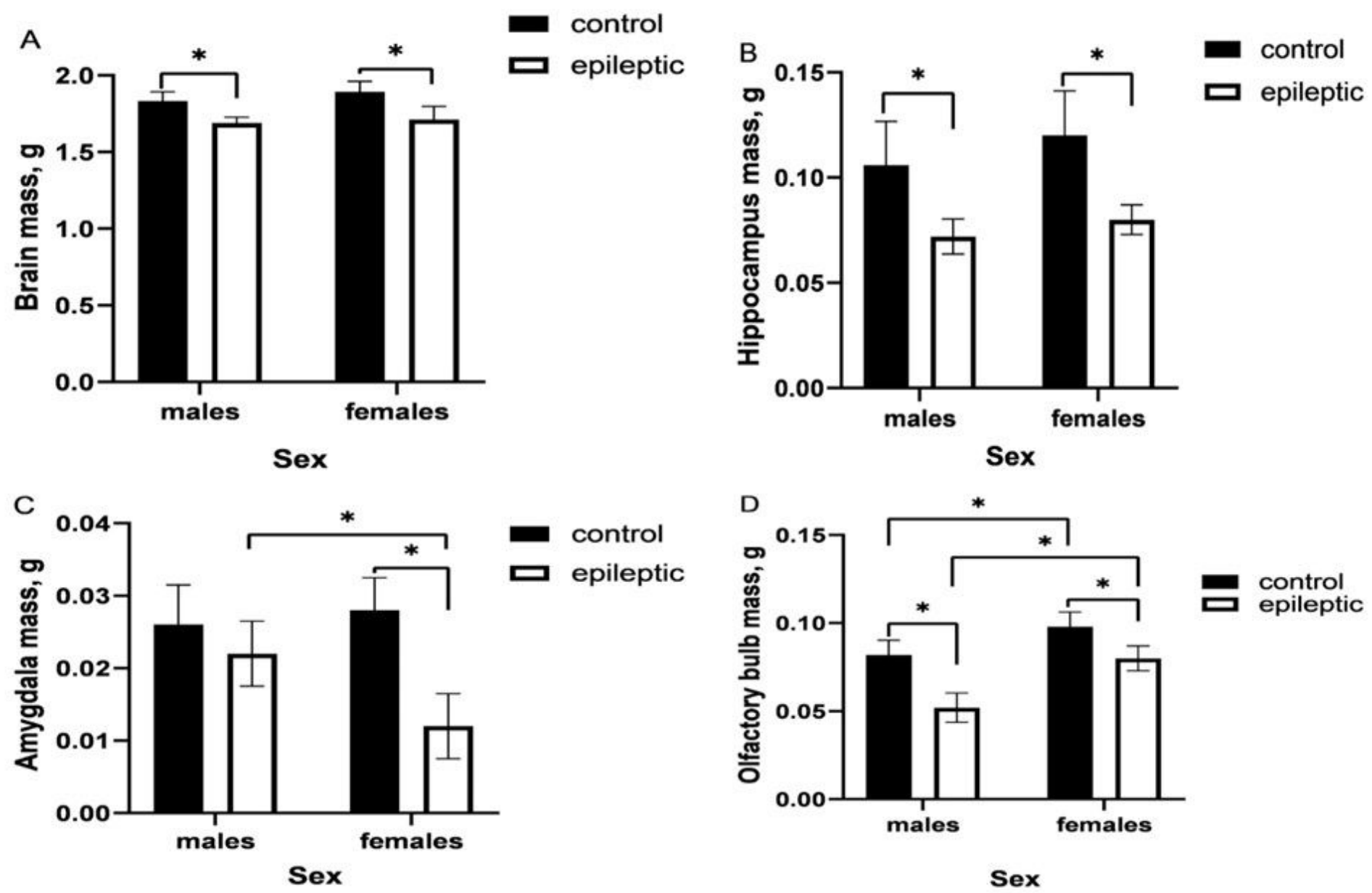

Figure 3

Mass changes (in grams) in the total brain (A), hippocampal formation (B), amygdala (C) and olfactory bulb (D) of male and female epileptic Wistar rats in comparison to respective control groups. ${ }^{\star} p<0.05$ for significance.
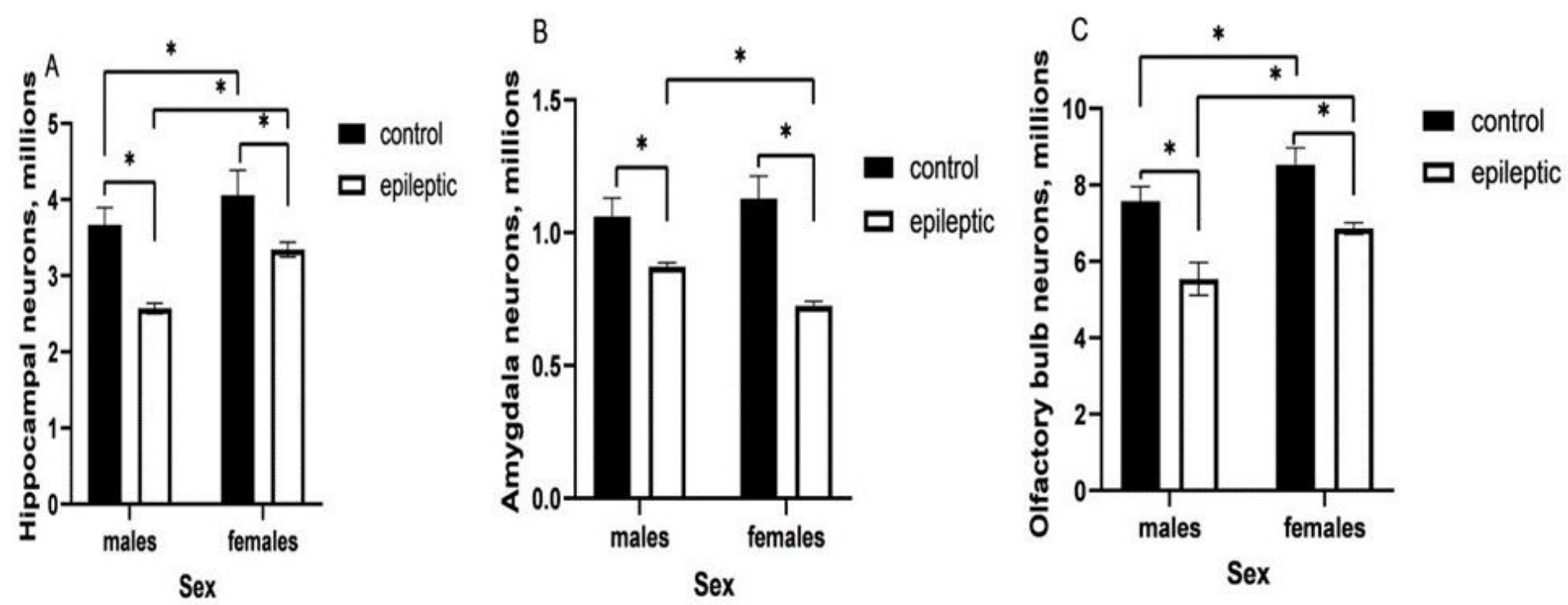
Figure 4

Number of neurons (in millions) in hippocampal formation (A), amygdala (B), and olfactory bulb (C) of male and female epileptic Wistar rats and respective controls. Data and level staples are expressed as mean \pm standard deviation. ${ }^{\star} p<0.05$ for significance.
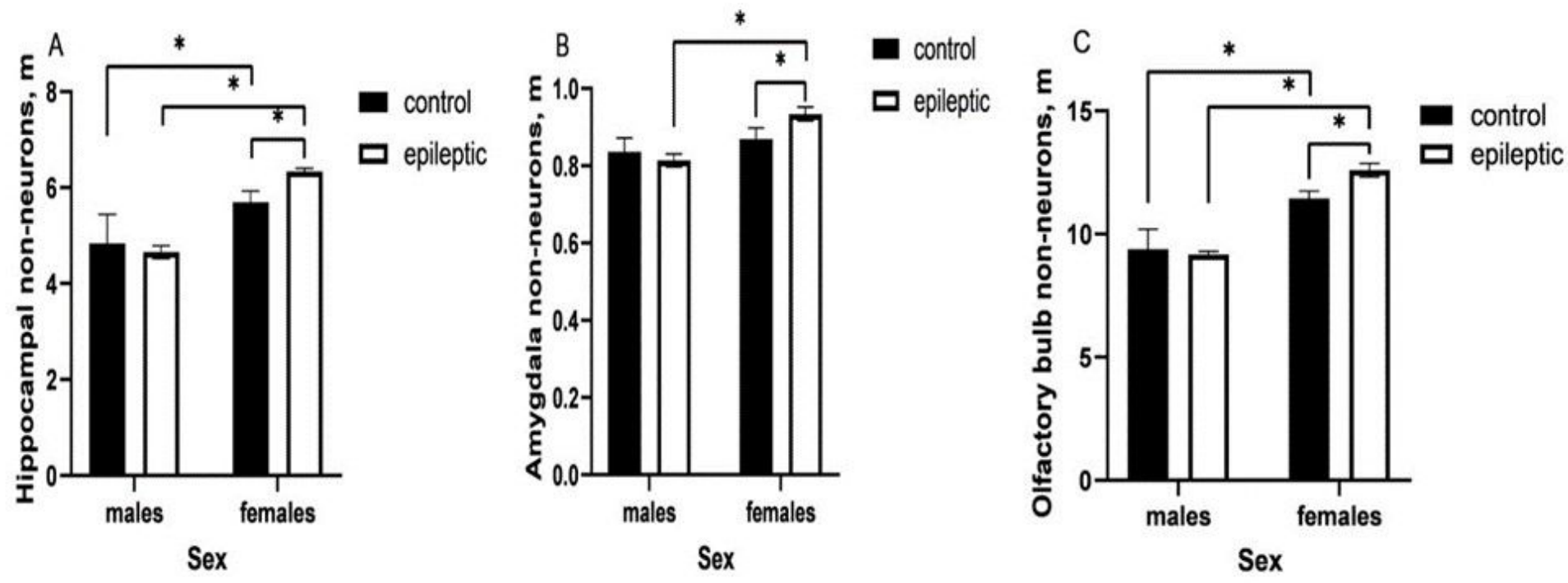

Figure 5

Number of non-neuronal cells (in millions) in hippocampal formation (A), amygdala (B), and olfactory bulb (C) of male and female epileptic Wistar rats and respective controls. Data and level staples are expressed as mean \pm standard deviation. ${ }^{*} \mathrm{p}<0.05$ for significance by two-way ANOVA and Tukey's posthoc test. 